\title{
Estudio Exploratorio de Factores que Influyen en la Decisión de la Mujer para Estudiar Ingeniería en Chile
}

\author{
Claudia A. Jiménez ${ }^{(1)}$, Eduardo A. Jones ${ }^{(1)}$, y Cristian L. Vidal(1, 2)* \\ (1) Ingeniería Civil Informática, Escuela de Ingeniería, Campus Rodelillo, Universidad Viña del Mar, Agua Santa 7055, \\ Viña del Mar-Chile (e-mail: cjimenez@uvm.cl; ejones@uvm.cl; cristian.vidal@uvm.cl) \\ (2) Escuela de Ingeniería Informática, Facultad de Ingeniería, Ciencias y Tecnología, Universidad Bernardo O’Higgins, \\ Avenida Viel 1497, Ruta 5 Sur, Santiago - Chile (e-mail: cristianvidal@docente.ubo.cl) \\ * Autor a quien debe ser dirigida la correspondencia
}

Recibido Ago. 13, 2018; Aceptado Oct. 26, 2018; Versión final Nov. 27, 2018, Publicado Ago. 2019

\section{Resumen}

Los principales objetivos de este trabajo son entregar detalles de un estudio exploratorio sobre las mujeres ingenieras en Chile y presentar resultados de la positiva realidad laboral en Chile de mujeres ingenieras. Las entidades chilenas de educación superior, ofrecen una amplia y variada propuesta de estudios superiores de ingeniería con diversas especialidades. A pesar del amplio rango de posibilidades de estudios en ingeniería, el número de mujeres estudiantes es mucho menor que los hombres, situación que no es común en otras carreras universitarias. Los resultados muestran la relevancia de la formación matemática y de ciencias tanto en la enseñanza básica y educación media para las mujeres que terminan exitosamente una carrera de ingeniería.

\section{Exploratory Study of Factors that Influence the Decision of Women to Study Engineering in Chile}

\begin{abstract}
The main objectives of this work are to provide details of an exploratory research about women engineers in Chile and thus present results of the favorable labor situation of women engineers in Chile. The Chilean institutions of higher education offer a wide and varied proposal of higher studies in engineering with several majors. Despite this range of possibilities of studies in engineering, the number of women students is much lower than that of men, a situation not common in other university careers. The results show the relevance of mathematics and science education in primary and secondary school for women who complete an engineering career.
\end{abstract}




\section{INTRODUCCIÓN}

La participación de las mujeres en las áreas de las Ciencias, Tecnología, Ingeniería y Matemáticas (CTIM en español, más conocido como STEM en inglés) es un tema ya estudiado en varios países del mundo y bajo distintos enfoques desde metodologías de análisis del discurso en estudiantes de ingeniería mujeres (Starovoytova y Cherotich, 2016) hasta estudios sociológicos relacionados con las razones de por qué las mujeres no ingresan o no terminan carreras en estas líneas (Mavriplis et al., 2010; White y Massiha, 2016). Tal y como lo subraya Puri (2018), aun cuando algunos campos de estudio han experimentado cambios significativos hacia una igualdad de género, las escuelas de ingeniería aún no encuentran la forma de superar el umbral del $20 \%$ de participación femenina. Respecto a esta situación en Chile, es necesario considerar que, las carreras de ingeniería, además de distinguirse por su orientación o especialidad, también se diferencian en su foco o profundidad respecto a su duración. Así, en Chile se distingue entre ingeniería de ejecución, ingeniería, e ingeniería civil con una duración usual de 4, 5 y 6 años respectivamente. Ya existen casas de estudios superiores en Chile que innovan en la duración habitual de las carreras de ingeniería con ingeniería a 4 años e ingeniería civil a 5 años (UVM, 2018). Según el Consejo Nacional de Educación (CNED, 2016), tal y como lo muestra la figura 1, la participación de mujeres en carreras de ingeniería (tecnología) en los años 2015 y 2016 en Chile era en torno al $20 \%$.

Según Ing2030 (2018), el aumento de mujeres en carreras de ingeniería no ha sido significativo en un lapso de 10 años: $20 \%$ el año 2004 y $24 \%$ el año 2014. Estas cifras no se condicen con las condiciones actuales referidas a la equidad de género, de inclusión y participación femenina en Chile, posibles de ver en otros entornos de la sociedad, como es el caso del emprendimiento femenino que es alrededor de un $40 \%$ (Arellano y Peralta, 2016). Esta baja participación de mujeres en ingeniería es un asunto a nivel global. La herramienta de análisis de brecha de género del Pacto Mundial (PactoMundial, 2018) busca ayudar a los líderes empresariales en el mundo a identificar fortalezas, debilidades y oportunidades de mejoras para lograr una igualdad en las condiciones laborales entre hombres y mujeres. Adicionalmente, también hay experiencias de empresas como Facebook y Linkedln (20Minutos, 2018) que usan y crean programas de asesoramiento a mujeres jóvenes que estudian ingeniería informática o computación como una forma de aumentar su interés en el tema como forma de incentivar el egreso y finalización de las carreras ya que en Estados Unidos existe una baja en el porcentaje de egreso femenino en dichas áreas: de un 35\% en 1985 a un $18 \%$ en 2015. De manera similar, en educación superior en Chile, hoy existen universidades que han tomado medidas para proporcionar cupos especiales en carreras de ingeniería para mujeres (PEG, 2018).

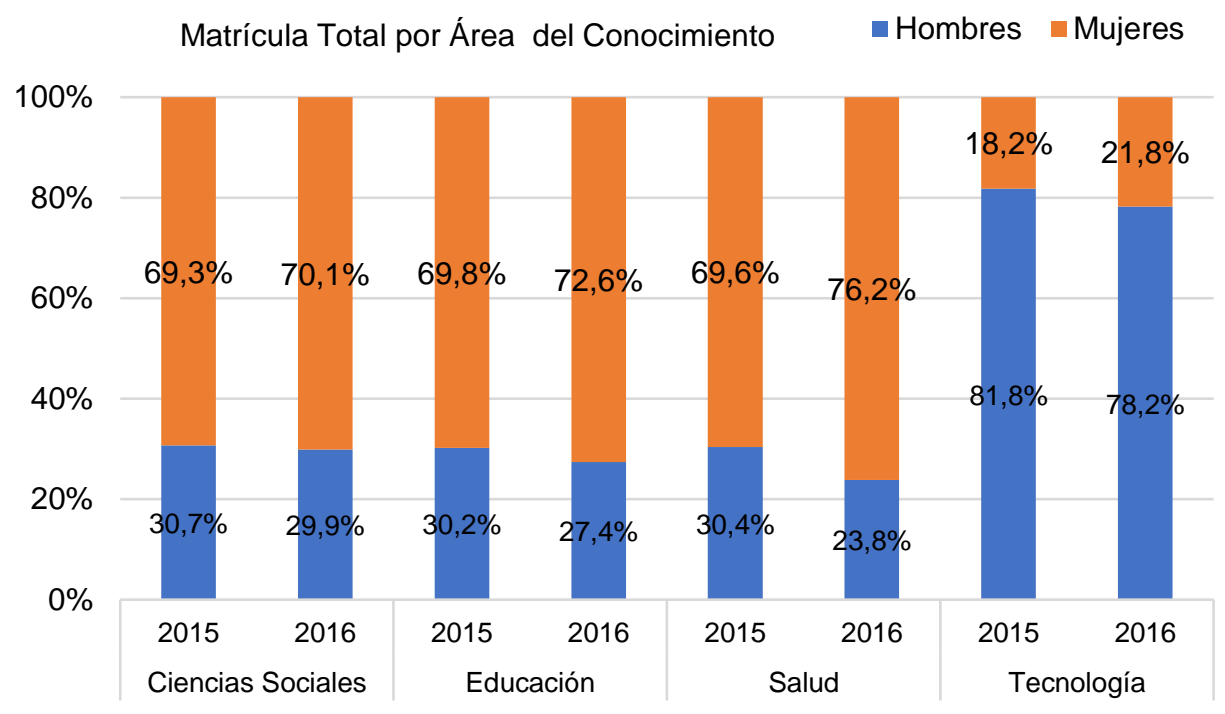

Fig. 1. Participación de mujeres en carreras de ingeniería años 2015 - 2016.

Las dos primeras filas de la tabla 1 (CNED, 2016), las áreas de ingeniería con una mayor participación femenina corresponden a ingeniería aplicada en sectores cercanos a la biología (ingeniería en alimentos y similares, e ingeniería biomédica). Sin embargo, esta diferencia no es realmente significativa respecto a la participación de hombres, como sí lo muestran las otras filas de la tabla 1 que representan el porcentaje de participación de hombres y mujeres en otras áreas de la ingeniería. Por ejemplo, en ingeniería mecánica la brecha entre hombres y mujeres es superior al $95 \%$ mientras que en ingeniería electrónica y similares tales como ingeniería informática y/o de computación esta brecha es superior al $94 \%$. Según (González-Palencia Jiménez y Jiménez, 2016), a pesar del alto desarrollo de las Tecnologías de la Información y la Comunicación (TIC), en dicha área la participación femenina sólo representa una tubería que gotea, es decir, muchas de 
ellas quizás fueron atraídas por el universo de la tecnología pierden dicho interés. En contraposición a esta baja participación, el sector industrial reconoce que las habilidades sociales, el liderazgo y la capacidad de trabajo en equipo son aspectos claves que caracterizan a estas profesionales, cualidades que son altamente valoradas (CNED, 2016).

Tabla 1: Lista de carreras del área Tecnología con una mayor participación de mujeres y hombres del año 2016.

\begin{tabular}{|l|c|c|c|}
\hline \multicolumn{1}{|c|}{ Carrera Genérica } & $\begin{array}{c}\text { \% Matricula } \\
\text { Total Hombres }\end{array}$ & $\begin{array}{c}\text { \% Matricula } \\
\text { Total Mujeres }\end{array}$ & $\begin{array}{c}\text { Brecha Ptos. } \\
\text { Porcentuales }\end{array}$ \\
\hline Ingeniería en alimentos y similares & $32,30 \%$ & $67,70 \%$ & 35,5 \\
\hline Informática biomédica & $40,20 \%$ & $59,80 \%$ & 19,5 \\
\hline Ingeniería en mecánica automotriz & $97,50 \%$ & $2,50 \%$ & 95,1 \\
\hline Ingeniería electrónica y similares & $97,30 \%$ & $2,70 \%$ & 94,5 \\
\hline Ingeniería en mantenimiento industrial y similares & $96,20 \%$ & $3,80 \%$ & 92,4 \\
\hline Ingeniería eléctrica y similares & $96,20 \%$ & $3,80 \%$ & 92,3 \\
\hline Ingeniería en automatización, control industrial y similares & $95,00 \%$ & $5,00 \%$ & 89,9 \\
\hline
\end{tabular}

En la búsqueda de causas y razones de la baja participación de mujeres en carreras de ingeniería en Chile, este artículo presenta un estudio exploratorio para conocer detalles acerca del grado de satisfacción y realidad laboral de mujeres ingenieras en Chile, y así contrastar dichos resultados con el bajo número de mujeres que estudian ingeniería en Chile en la actualidad, con el fin de determinar patrones comunes y así establecer una base de información en la búsqueda de mejorar dichos resultados. Específicamente, este trabajo pretende responder las siguientes preguntas: ¿Qué determina que una joven decida estudiar ingeniería?, ¿Cuáles son las variables que inciden en su decisión?, y ¿Son comunes estas variables en las estudiantes y mujeres ingenieras? Como menciona Tierjen (2017), existen contribuciones fascinantes de mujeres en las áreas de la ciencia y la ingeniería en la historia del mundo. Desde Xilingshi en China quien dirigió el desarrollo del cultivo de la seda y el tejido industrial; Cleopoatra, una alquimista, quién probablemente vivió en Alejandría, Egipto. Hoy, el trabajo de los alquimistas es reconocido como el precursor de la química moderna, y Cleopatra estaba interesada en los pesos y las medidas, e ilustró los primeros dibujos de sustancias químicas; hasta Ada Lovelace, quien vivió desde 1815 a 1842 (hoy existe un lenguaje de programación en su honor), se considera la primera persona en describir la programación de computadores.

Según National Research Council (2010), por un estudio del Instituto Politécnico de Massachussets (más conocido como MIT por sus siglas en inglés), en el año 1999 ya existía preocupación por las barreras que enfrentaban las mujeres en las facultades de ciencias e ingeniería en las universidades del mundo: Si bien existía un creciente aumento de las mujeres en ingeniería en universidades del mundo, este número correspondía a una muy baja representación respecto a la participación de los hombres, una realidad similar actualmente en diversos lugares tal como en España donde sólo un $25 \%$ de los estudiantes de ingeniería son mujeres (ElConfidencial, 2018). En esta línea, Catalyst (2018) destaca que las mujeres en el mundo empleadas en investigación y desarrollo (I + D) el año 2014 sólo representaron menos de un tercio $(28,8 \%)$ del total de las personas empleadas en dicha área. Según Wes (2018), en Inglaterra, en el año 2017, el 11\% de la fuerza de trabajo de ingeniería es femenina lo que representa un cambio positivo respecto al $9 \%$ en 2015. Tal y como señala De Souza y Ney (2017), aún existen muchas barreras para una mujer en la ingeniería comúnmente de hombres, y las mujeres ingenieras son ejemplos de quienes sobreviven en un entorno esencialmente masculino. Considerando los focos o niveles de profundidad de las carreras de ingeniería en Chile, y considerando, el área de ingeniería y Negocios como un área especial de la ingeniería en Chile, de acuerdo con los resultados de ENSI (2017) acerca de la participación laboral en ingeniería en Chile con carreras tales como ingeniería comercial e ingeniería en administración de empresas cuya duración es usualmente de 9 a 10 semestres académicos, la figura 2 muestra que la participación femenina en ingeniería es de un $18 \%$ y la mayor participación de las mujeres es en el área de ingeniería y negocios.

\section{METODOLOGIA}

A continuación, se presentan detalles de este estudio y sus resultados, para el análisis de estos y una comparación con los resultados de ENSI (2018). Este estudio fue realizado durante 2017 mediante una plataforma en línea, previo contacto con un universo de 537 egresadas de carreras del área de ingeniería de instituciones de educación superior en Chile, de las cuales se obtuvo un total de 129 participantes. 

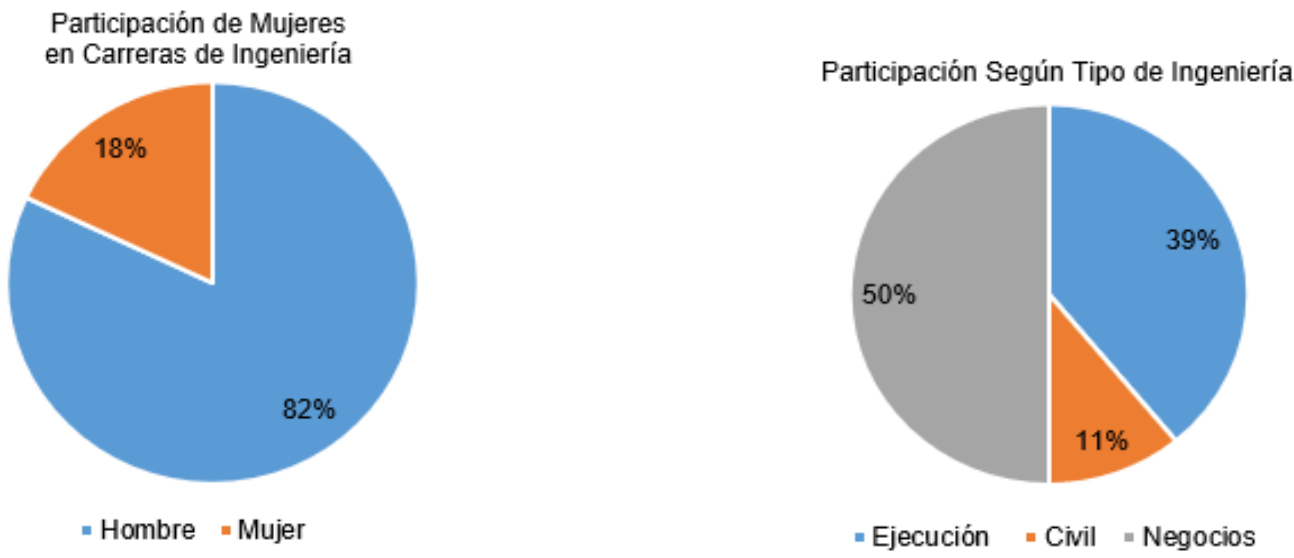

Fig. 2: Participación de mujeres en ingeniería en Chile y distribución según nivel de profundidad.

\section{Encuesta}

Las preguntas de este estudio se clasifican en Datos Generales (16 preguntas), Características personales de la vida pre-universitaria de la encuestada (7 preguntas), Características de la vida de la encuestada en la enseñanza básica (15 preguntas), Características de la vida de la encuestada en la enseñanza media (18 preguntas), Autoconcepción y motivación de la encuestada (13 preguntas), y Vida preuniversitaria - Elección de carrera (11 preguntas), es decir, la encuesta está formada de 80 preguntas.

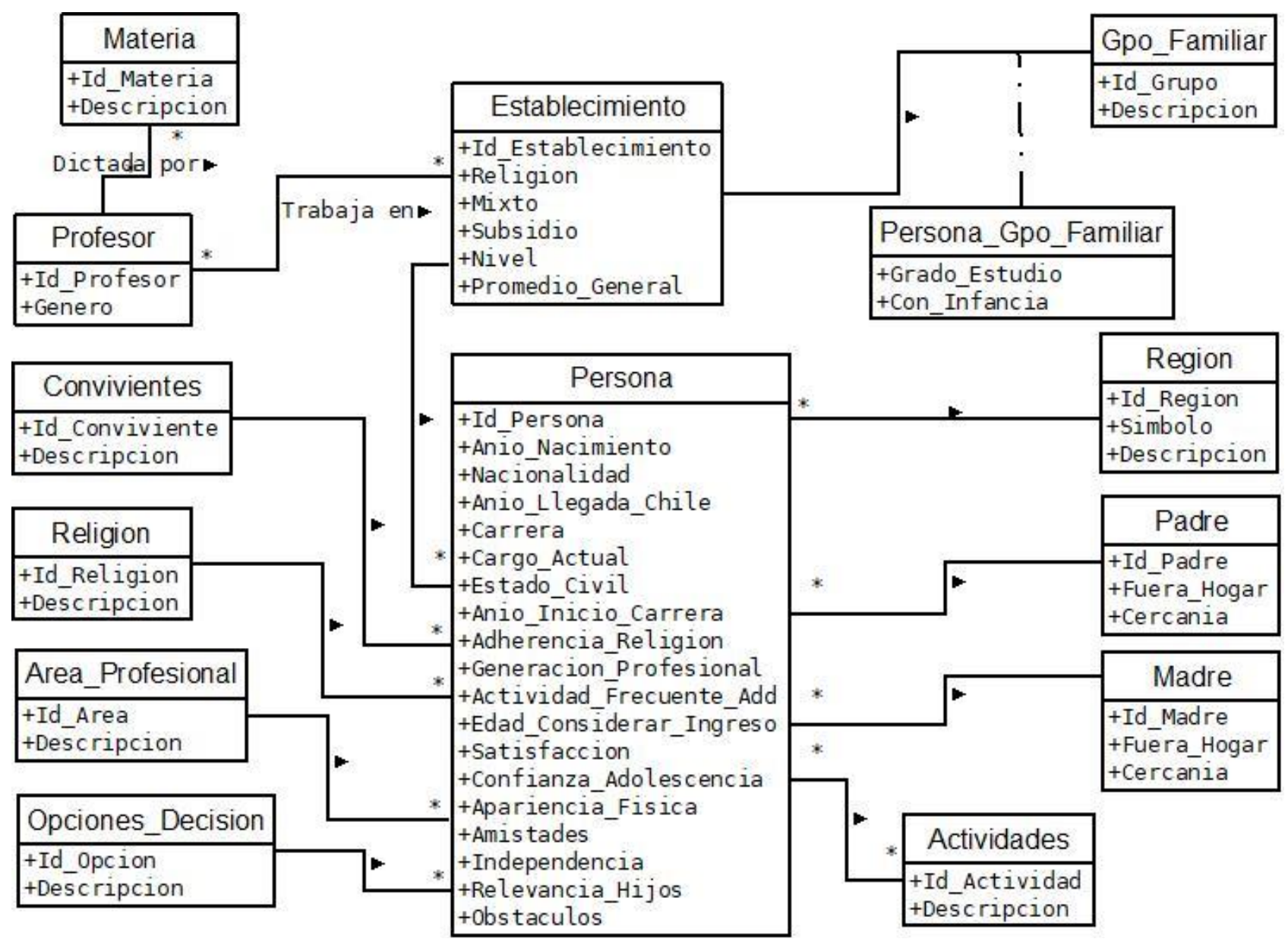

Fig. 3: Modelo de datos para encuesta de estudio.

La figura 3 muestra a nivel conceptual un modelo conceptual (diagrama de clases UML) con detalle solamente de los atributos de cada una de las entidades (clases) que son la base de modelo de datos de la base de datos para la información de este estudio. La clase principal de esta figura corresponde a Persona la cual 
presenta diversos atributos y relaciones con otras clases, todas ellas relevantes en este estudio tales como los atributos Satisfacción y Generacion_Profesional junto con las clases Establecimiento, Materia y Profesor las cuales se refieren a la formación educacional previa.

\section{RESULTADOS}

Se propuso conservar la mayor cantidad de datos de la encuesta original posibles con el objetivo de que el punto de vista de las mujeres que han estudiado alguna carrera involucrada con la ingeniería pueda ser reflejado de la forma más clara posible. Para la integridad de los resultados en cuanto a gráficos se refiere, se han tomado en cuenta solamente las respuestas de las personas que han concluido la encuesta anteriormente presentada, lo que pasa a ser un $80 \%$ de la población total encuestada, es decir una cifra muy significativa.

Dentro de los resultados más significativos se destaca: i) La especialidad más elegida por las mujeres ingenieras es la ingeniería civil y por el otro lado, la menos elegida es la ingeniería en ejecución; ii) Las mujeres ingenieras en la actualidad pertenecen en su mayoría a la primera generación de profesionales de su familia; iii) El grado de satisfacción de la elección de la carrera es muy superior a la opción de haber elegido otra opción de formación profesional para las mujeres ingenieras; iv) De igual forma, las encuestadas han respondido que, de haber un profesor involucrado en su decisión de estudiar una ingeniería, dicho profesor(a) sería del área de matemáticas; v) De las mujeres ingenieras que en la actualidad practican su profesión, la mayor parte se encuentra en un puesto de jefatura; vi) La religión que predomina entre las encuestadas es la católica; vii) Los familiares de gran parte de la población encuestada no han alentado a la elección de carrera.

Se efectuó además un cruce de datos para el que se ha tomado énfasis en: i) Promedio de matemáticas en educación básica vs educación media junto con la relevancia de los profesores en sus asignaturas según áreas de formación; ii) Juegos en la infancia (actividades extracurriculares); iii) Apoyo familiar de la elección de carrera; iv) Motivación familiar para la elección de una carrera de ingeniería; v) Relevancia entre generación profesional en la familia vs apoyo familiar por la carrera escogida; vi) Nivel de subjetividad de reconocimiento y/o exigencia académica; vii) Relevancia de género de las personas influyentes en la elección de carrera; viii) Actividades extracurriculares en la enseñanza media; ix) Gráficos de autopercepción en la adolescencia; $x$ ) Actividades realizadas frecuentemente con amigos.

Las figuras 4 y 5 muestran resultados significativos de este estudio para las 3 primeras variables mencionadas. Específicamente, la figura 4 ilustra el porcentaje de elección para mujeres del tipo de ingeniería según su nivel de profundización (civil, ejecución u otra), además de la distribución porcentual de las mujeres en la generación de profesionales en sus familias.

\section{Elección del Tipo de Ingeniería}

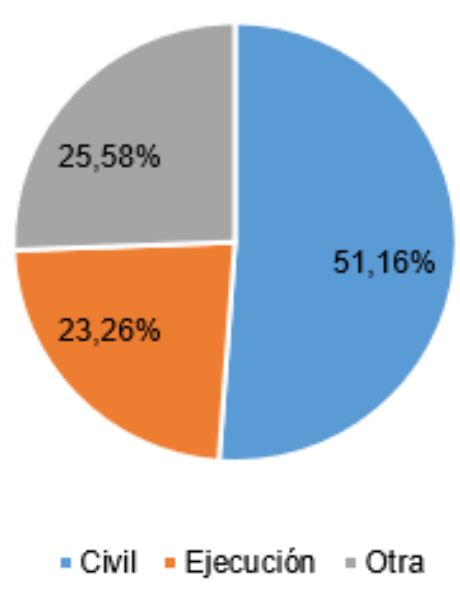

\section{Generación Profesional en la Familia}

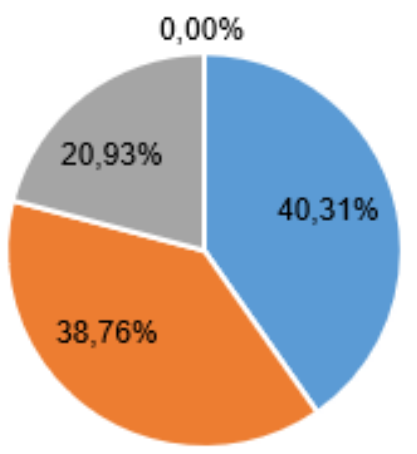

- 1era Generación

- 3era Generación

\section{- 2da Generación \\ - 4ta Generación o Superior}

Fig. 4: Porcentajes de elección entre las opciones de profundización de mujeres en carreras de ingeniería.

La figura 5 presenta el grado de satisfacción de mujeres que eligieron ingeniería en su formación profesional y el porcentaje de ellas dispuestas a elegir otra formación profesional si es que eso fuera posible. La figuras 6 detalla el porcentaje de los rendimientos de calificaciones promedio en matemáticas en la enseñanza básica versus y las calificaciones promedio en matemáticas en la educación media, mientras que las figuras 7 y 8 muestran la relevancia de los profesores de las asignaturas según el área de conocimiento tanto en educación básica como enseñanza media, respectivamente, en los cuales se aprecia la relevancia de la educación matemática y el papel para la elección de formación profesional. 


\section{DISCUSIÓN}

Según el primer grafico de la figura 4 , sobre el $50 \%$ de las mujeres del estudio son ingenieras civiles, aun cuando el segundo gráfico de la figura 2 señala que un mayor porcentaje de mujeres elije ingeniería del área de negocios o ingeniería de ejecución, lo que puede indicar que es alto el rango de mujeres que estudian ingeniería civil y finalizan exitosamente sus estudios. Los resultados que presenta la figura 5 reafirman esta idea, ya que existe un alto grado de satisfacción de mujeres ingenieras en su formación profesional respecto al porcentaje de mujeres dispuestas a elegir otra formación profesional, aun cuando hay un alto porcentaje de mujeres ingenieras que, aunque están satisfechas con su elección, estarían dispuestas a elegir otra opción de formación. Una de las razones, tal como lo señala McGregor et al. (2017), podría ser la brecha salarial que existe entre mujeres y hombres en ingeniería.

Los cruces de datos de las figuras 6, 7 y 8 ratifican el alto grado de afinidad para con las matemáticas de las mujeres en ingeniería en Chile. Como muestra la figura 6, en un alto grado porcentual, en Chile las mujeres en ingeniería presentan calificaciones promedio entre 6 y 7 (en Chile, la calificación o nota mínima es 1 mientras que la calificación máxima es 7), y sus profesores o profesoras de matemáticas tanto en educación básica (figura 7) como enseñanza media (figura 8) jugaron un papel relevante en la elección de ingeniería como opción de formación profesional. Así, para responder las preguntas ¿Qué determina que una joven decida estudiar ingeniería?, ¿Cuáles son las variables que inciden en su decisión? y ¿Son comunes estas variables en las estudiantes y mujeres ingenieras?, con los resultados de este estudio, queda claro que la formación matemática, así como el rol del profesor o profesora de matemáticas en los estudios previos a la universidad son factores altamente determinantes y comunes para las mujeres ingenieras para su elección de formación.

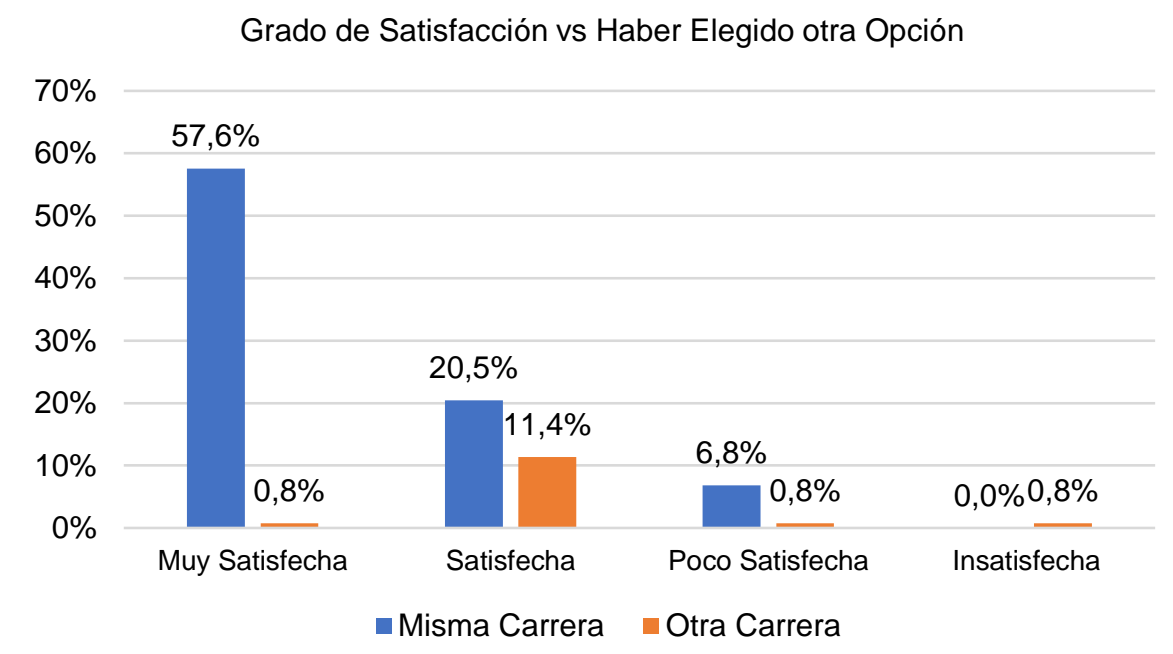

Fig. 5: Primeros resultados significativos de estudio de las causas de la participación baja de mujeres en ingeniería.

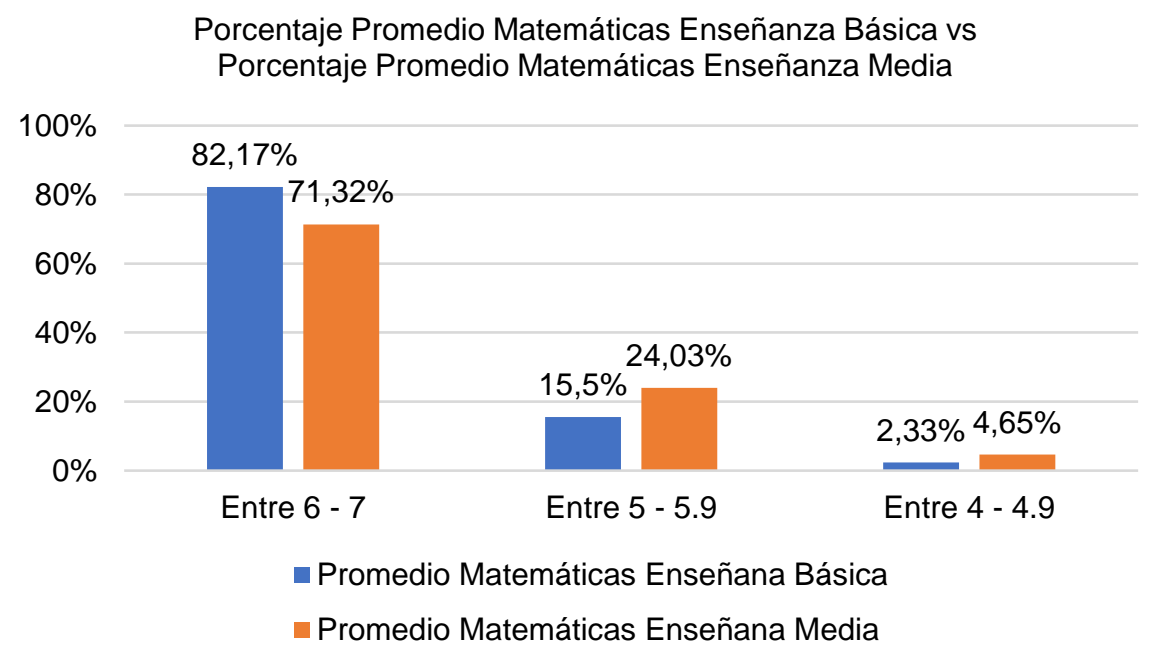

Fig. 6: Porcentaje de promedios de notas de matemáticas en enseñanza básica vs enseñanza media de mujeres ingenieras. 
Los resultados previamente descritos reafirman ideas de trabajos previos y en otros lugares del mundo (González-Palencia Jiménez y Jiménez, 2016; Hunt, 2016; Corbett, 2015) respecto a las barreras de las mujeres en estudios de ingeniería, aun cuando Hunt (20106) indica que una razón principal son los sueldos y asensos laborales futuros (una razón no plausible en Chile). Corbett (2015) además considera otras variables que afectan a la mujer en el área de la ingeniería como el sexismo y el acoso sexual.

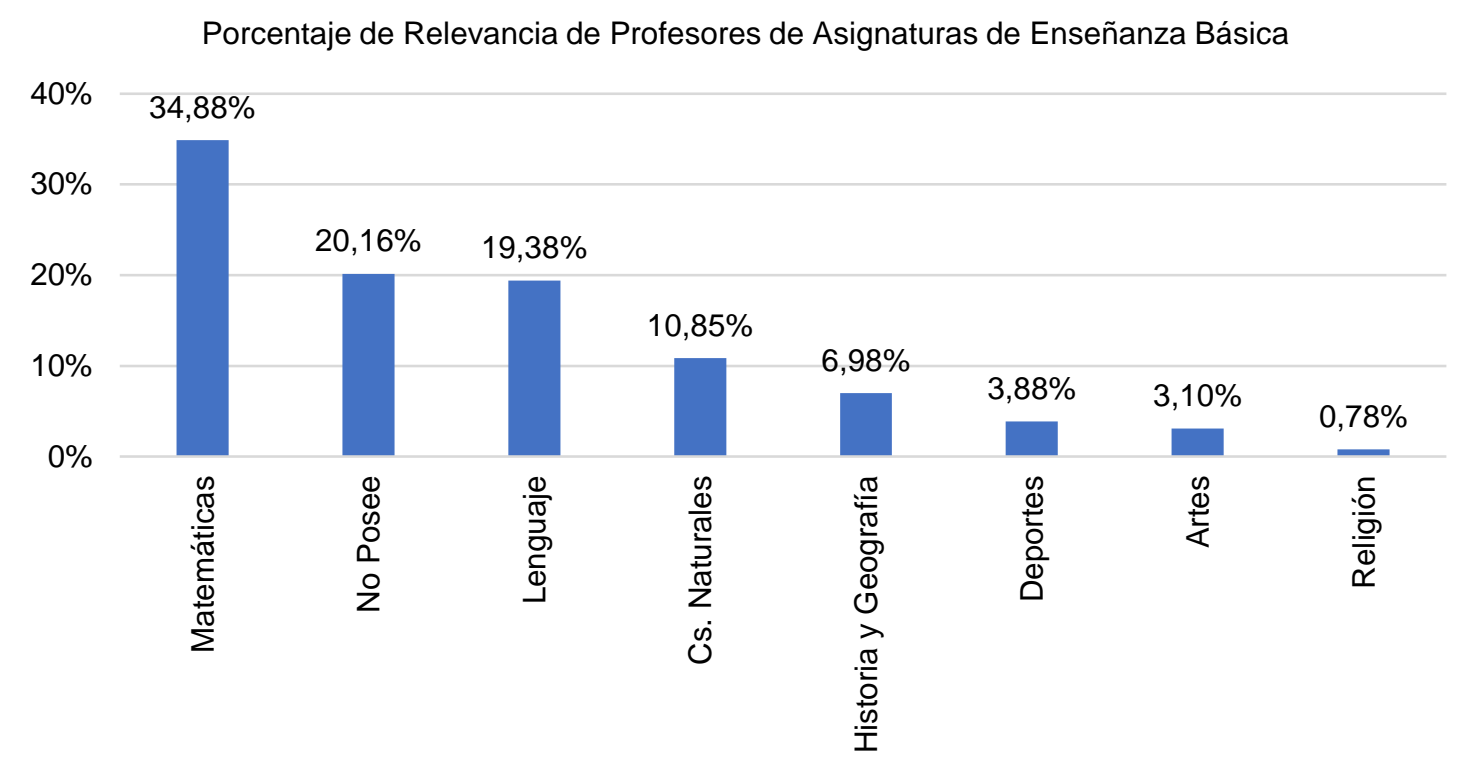

Fig. 7: Porcentaje de relevancia de profesores de asignaturas en educación básica de mujeres ingenieras.

Porcentaje de Relevancia de Profesores de Asignaturas de Enseñanza Media

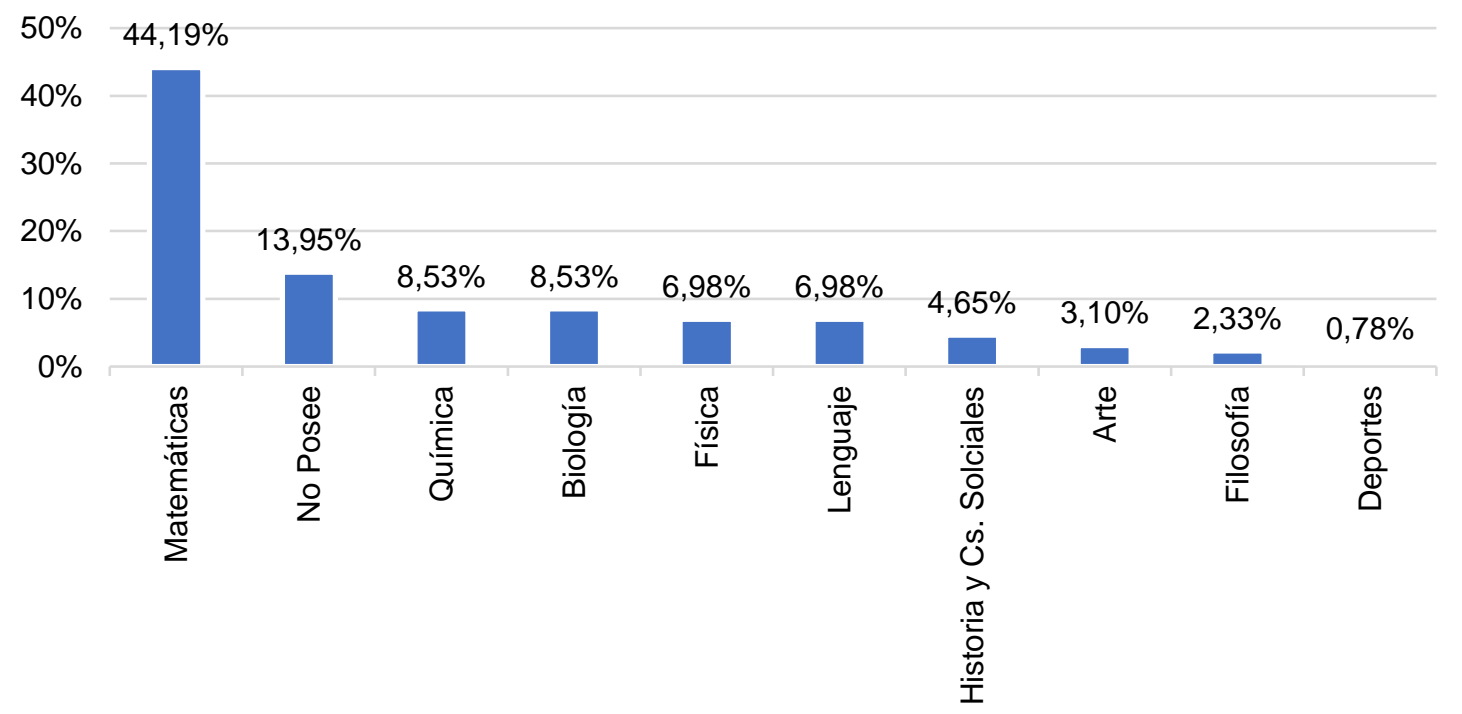

Fig. 8: Porcentaje de relevancia de profesores de asignaturas en enseñanza media de mujeres ingenieras.

\section{CONCLUSIONES}

Ingeniería es una profesión considerada para hombres desde sus inicios, pero actualmente la mujer también se interesa por carreras de ingeniería. Sin embargo, tal como presentó este estudio, la distribución por sexos de matriculación en carreras de ingeniería en Chile es muy similar a la de tiempo atrás lo que evidencia un estancamiento en el interés de las mujeres por seguir estudios de ingeniería.

Buscar la equidad entre hombres y mujeres resulta muy importante en la actualidad y que los posibles prejuicios desaparezcan, ya que una gran cantidad de estudios, incluyendo este trabajo, demuestran que las capacidades de hombres y mujeres para con las matemáticas, tecnología y ciencias pueden ser iguales y la diferencia no tiene nada que ver con el género del estudiante. Es viable obviar y hasta eliminar la pregunta ¿Por qué las mujeres no quieren ser ingenieras? 
Con los resultados de este trabajo se puede afirmar que en Chile la formación de matemáticas y ciencias junto con la afinidad con los profesores de dichas asignaturas tanto en la educación básica (primaria) y media (secundaria) corresponden a factores determinantes para las mujeres en la decisión de su formación profesional en estudios de ingeniería.

Esto es una invitación para el sistema Educacional en Chile a invertir en la formación y preparación de profesores en las áreas de matemáticas y ciencias de nivel tanto para educación básica como media, lo que es de absoluta relevancia actualmente en Chile y así lograr una educación no sexista en todas las áreas de formación.

\section{REFERENCIAS}

20Minutos., Facebook y Linkedln se alían para respaldar a las mujeres en ingeniería e informática (2018)

Arellano, S. y S. Peralta, Informe de Resultados: Emprendimiento y Género, Cuarta Encuesta de MicroEmprendimiento, Ministerio de Economía, Fomento y Turismo, Gobierno de Chile, Marzo (2016)

Catalyst., Women in Science, Technology, Engineering and Mathematics (STEM) (2018)

CNED., Consejo Nacional de Educación, Índices 2016 - Tendencias Matrícula Educación Superior (2018)

Corbett, C., Solving the Equation: The Variables for Women's Success in Engineering and Computing, ISBN: 9781879922457, AAUW, 33 - 46 (2015)

De Souza, M.B. y F. R. Ney, Every Female Engineer is a Survivor - A Study of Defense Strategies in the Work of Female Engineers, doi: 10.13189/ujm.2017.050402, Universal Journal of Management, 5 (4), 175-180 (2017)

ElConfidencial., Por qué cada vez menos mujeres en España quieren ser ingenieras (2018)

ENSI., Estudio Nacional de Sueldos de Ingenieros 2017 - Quinto Estudio Anual (2018)

González-Palencia Jiménez, R. y C. Jiménez Fernández, La brecha de género en la educación tecnológica, doi: 10.1590/S0104-403620160003000010, en Ensaio: Avaliação e Políticas Públicas em Educação, 24 (92), $743-771$ (2016)

Hunt, J., Why do Women Leave Science and Engineering? doi: 10.1177/0019793915594597, ILR Review, 69(1), 199226 (2016)

Ing2030., Ingeniería 2030 (2018)

Mavriplis, C., R. Heller y otros cinco autores, Mind the Gap: Women in STEM Career Breaks, doi: 10.4067/S071827242010000100011, Journal of Technology Management \& Innovation, Universidad Alberto Hurtado, Facultad de Economía y Negocios, Chile 5 (1), 140- 151 (2010)

McGregor, J., S. Graham, L. S. Giddings y J. Pringle, Pursuing equal pay: The perspectives of female engineers and potential policy interventions, doi: 10.1177/0022185616659677, Journal of Industrial Relations, SAGE Publications Ltd, 59 (1), 3 - 21 (2017)

National Research Council., Gender Differences at Critical Transitions in the Careers of Science, Engineering, and Mathematics Faculty, doi: 10.17226/12062, The National Academies Press, Washington, USA (2010)

PactoMundial., Nueva herramienta del Pacto Mundial para implementar la igualdad de género en las empresas (2018)

PEG., Programa de Ingreso Prioritario de Equidad de Género (PEG) (2018)

Puri, I., Why Engineering Schools Globally Need More Women, Institute of Public Works Engineering Australasia Queensland, International Women's Day Feature Articles, 32-33, Marzo (2018)

Starovoytova, D. y S. Cherotich, Challenges Faced by Female-Students in Engineering-Education, doi: 10.1037/00223514.66.2.268, Journal of Education and Practice, 7 (25) (2016)

Tierjen, J.S., Engineering Women: Re-visioning Women's Scientific Achievements and Impacts, ISBN 3319407988, Springer, $1^{\text {st }}$ Ed. (2017)

UVM., Escuela de Ingeniería (2018)

Wes., Statistics on Women in Engineering (2018)

White, J. y G. H. Massiha, The Retention of Women in Science, Technology, Engineering, and Mathematics: A Framework for Persistence, International Journal of Evaluation and Research in Education (IJERE), 5 (1), 1 - 8, Marzo (2016). 